\title{
Helping your child with separation anxiety disorder
}

\section{Opinion}

One of the more frustrating and confusing dilemmas of parenthood occurs when children's path towards independence is thwarted by separation issues. Fears, anxiety, or apprehension over some dreaded experience grips children's will, interfering with their ability to assume the normal expectations for their age. Sleeping by themselves, sleepovers at a friend's house sleep away camps, or other opportunities that entail overnights away from home, are passed up. Parents vacillate between worry and exasperation as they watch their children persistently avoid the steps that are so important to future emotional independence.

\section{Strategies to help with child separation anxiety or fear of leaving home}

\section{Consider the possible roots of the problem}

Children who suffer from separation troubles have experienced some developmental challenge they were unable to master. The birth of a sibling, serious illness/injury of a parent, forced attendance at overnight camp, traumatic life experience, or some other disturbing event has partially pushed them off the path of emotional selfsufficiency. To be away from home leaves them feeling unanchored and adrift among anxiety and worry. Parents are wise to use this knowledge to empathically connect with their child's emotional state.

Use reassurance and reasoning when discussing this subject. Parents are urged to walk a fine line between comforting the clinging child and encouraging independence

Tipping too much in either direction will sabotage efforts to help your child successfully separate. Consider the following: "We understand you have trouble spending nights away from home. It seems like worry and uncertainty are strong and hard to overcome. But we know you notice how other kids your age are doing these things and having more fun in their life. We want this for you, too."

\section{Urge them to expose the fearful or unrealistic thinking that supports their avoidance}

Children with this problem tend to be bombarded by disturbing thoughts or images when the prospect of separation arises. These cognitions reinforce the wish to keep things familiar and take no emotional chances. Encourage them to talk about these thoughts and
Volume 9 Issue 2 - 2018

\author{
Steven Richfield \\ Clinical Psychologist, Philadelphia, USA
}

Correspondence: Steven Richfield, Clinical Psychologist, Philadelphia, USA, Tel 610-238-4450;

Email director@parentcoachcards.com

Received: March 29, 2018 | Published: April 26, 2018

guide them to a more appropriate examination of the worries rather than the extreme version occupying their mind.

\section{Offer both a self-soothing message and a means to gradually combat their fear}

If leaving home to be with non-family members is to feel safe, children must learn how to regard it that way

Explain how they can develop a calmer mind by reminding themselves of the freedom, fun, and security they have experienced outside of the home. Encourage them to think of this as a safety net they carry in their mind whenever the worried thinking appears. Gently prod them to take small separation steps that they have avoided in the past. Document their success on paper, so they can see the progress underway. Review the mental and emotional experience they went through and troubleshoot the obstacles they faced.

\section{Acknowledgements}

None.

\section{Conflict of interest}

The author declares there is no conflict of interest. 